\title{
Genetics of vaccination-related narcolepsy
}

Hanna M. Ollila ${ }^{1,2,3 *}$, Annika Wennerstrom ${ }^{3} *$, Markku Partinen ${ }^{4}$, Emmanuel Mignot $^{1}$, Janna Saarela $^{2}$,Turkka Kirjavainen ${ }^{5}$, Christer Hublin ${ }^{6}$, Logan D. Schneider ${ }^{1}$, Sari-Leena Himanen ${ }^{7}$ Outi

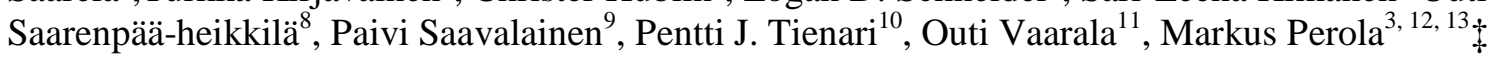

1. Stanford University, Center for Sleep Sciences and Medicine, Department of Psychiatry and Behavioral Sciences, Palo Alto, 94304, CA USA

2. Institute for Molecular Medicine Finland, University of Helsinki, Helsinki, Finland

3. National Institute for Health and Welfare, Helsinki, Finland

4. Helsinki Sleep Clinic, Vitalmed Research Center, Helsinki, Finland

5. Department of Pediatrics, Children's Hospital, Helsinki, Finland

6. Finnish Institute of Occupational Health, Helsinki, Finland

7. Department of Clinical Neurophysiology, Tampere University Hospital, Tampere, Finland

8. Department of Pediatrics, Tampere University Hospital, Tampere, Finland

9. Research Programs Unit, Immunobiology, University of Helsinki, Helsinki, Finland and Department of Medical and Clinical Genetics, University of Helsinki, Helsinki, Finland

10. Research Programs Unit, Molecular Neurology, Biomedicum, University of Helsinki, Helsinki, Finland; Department of Neurology, Helsinki UniversityHospital, Helsinki, Finland

11. Research Programs Unit, Diabetes and Obesity, University of Helsinki, Helsinki, Finland

12. University of Helsinki, Institute for Molecular Medicine, Finland (FIMM) and Diabetes and Obesity Research Program

13. University of Tartu, Estonian Genome Center, Tartu, Estonia

* Equal contribution

\$Correspondence to

Hanna M. Ollila

31665 Porter drive

CA 94304 Palo Alto, USA

Phone: +1 (650) 485-9647

Email:hannao@stanford.edu; hanna.m.ollila@thl.fi

Professor Markus Perola

National Institute for Health and Welfare,

PL 104, Haartmaninkatu 8, Biomedicum Helsinki

Helsinki 00251, Finland;

Phone: +358 295248727

Email: markus.perola@thl.fi 


\begin{abstract}
Narcolepsy type 1 is a severe hypersomnia affecting $1 / 3000$ individuals. It is caused by a loss of neurons producing hypocretin/orexin in the hypothalamus. In 2009/2010, an immunization campaign directed towards the new pandemic H1N1 Influenza-A strain was launched and increased risk of narcolepsy reported in Northern European countries following vaccination with Pandemrix ${ }^{\circledR}$, an adjuvanted H1N1 vaccine resulting in $\sim 250$ vaccination-related cases in Finland alone. Using whole genome sequencing data of 2000 controls, exome sequencing data of 5000 controls and HumanCoreExome chip genotypes of 81 cases with vaccination-related narcolepsy and 2796 controls, we, built a multilocus genetic risk score with established narcolepsy risk variants. We also analyzed, whether novel risk variants would explain vaccine-related narcolepsy. We found that previously discovered risk variants had strong predictive power (accuracy of $73 \%$ and $P<2.2 * 10^{-16}$; and ROC curve AUC 0.88) in vaccine-related narcolepsy cases with only $4.9 \%$ of cases being assigned to the low risk category. Our findings indicate genetic predisposition to vaccine-triggered narcolepsy, with the possibility of identifying $95 \%$ of people at risk.
\end{abstract}

Narcolepsy is a severe hypersomnia affecting 1/3000 individuals ${ }^{1-3}$. Narcolepsy type 1 (NT1) is characterized clinically with excessive daytime sleepiness and presence of cataplexy. It is irreversible and caused by a specific loss of hypocretin/orexin producing neurons in the lateral hypothalamus ${ }^{4}$. Patients with narcolepsy type 2 do not have unambiguous cataplexy and their CSF hypocretin/orexin levels are normal; whereas the hypocretin-deficient NT1 is theorized to be autoimmune-related. In 2009/2010 a vaccination campaign towards pandemic H1N1 Influenza-A was launched. Soon after, an increase in the incidence of narcolepsy was seen in Finland and in Sweden with 6.3/100,000 narcolepsy incidence in children, an 8-fold increase compared to the $0.79 / 100,000$ baseline incidence ${ }^{5}$. The increase was traced back to the use of Pandemrix ${ }^{\circledR}$ for H1N1 immunization ${ }^{5-11}$. Increased incidence of narcolepsy was subsequently detected in all countries where Pandemrix ${ }^{\circledR}$ was used; whereas countries using other brands did not see increases in narcolepsy incidence ${ }^{12,13}$. Other environmental risk factors that have been involved in triggering narcolepsy include H1N1 itself and Streptococcus pyogenes infections ${ }^{14-16}$.

The absence of hypocretin neurons in narcolepsy and its association with Influenza-A immunization/infection suggest an autoimmune origin for narcolepsy type 1 . This hypothesis is also supported by genetic risk factors that have been identified through Genome Wide Association Studies (GWAS) in narcolepsy. Indeed, nearly all individuals (98\%) with narcolepsy type 1 carry the exact same HLA DQBI*06:02 allele ${ }^{17-19}$ and HLA associations are seen in nearly all autoimmune diseases. Furthermore, variants within the T cell receptor (TRA and TRB) loci associate with narcolepsy both with and without vaccination as a trigger ${ }^{20-22}$. TCRs are expressed on T lymphocytes and carry out effector function by interacting with HLA molecules presenting specific epitopes, suggesting a specific antigen presentation pathway for the development of narcolepsy ${ }^{21}$. Similarly, other known genetic variants (CTSH, P2RY11, ZNF365, IFNAR1 and TNFSF4) interact with the same antigen presentation pathway or predispose to autoimmune diseases ${ }^{20-23}$.

Finally, many of the genetic effects seen in narcolepsy, such as those for HLA and with TRA, have unusually high effect sizes with OR $>8$ and 1.5 , respectively. Furthermore, both alleles show association with vaccination-related narcolepsy in Swedish individuals ${ }^{24}$. Due to the high effect sizes of known variants, we decided to examine, whether individuals with vaccine-related narcolepsy harbored known predisposing risk factors, and whether there were previously undiscovered genetic risk factors specific for vaccine-related narcolepsy cases. 


\section{Materials and Methods \\ Patients}

The diagnosis of narcolepsy type 1 (ICD-10 code G47.4) was based on criteria of the International Classification of Sleep Disorders (version 3, ICSD-3). In addition, all patients had overnight polysomnography and a positive Multiple Sleep Latency Test (i.e. $<8$ mins, with 2 or more REM sleep onsets). Absence of hypocretin in cerebrospinal fluid was confirmed in the majority of cases with lumbar puncture $(\mathrm{N}=58)$. Informed consent in accordance with governing institutions was obtained from all subjects and reviewed by ethical review boards of Helsinki University, Helsinki-Uusimaa Health District Ethical committee, and Stanford University. The study fully complies with national and international regulations regarding privacy and data protection relevant to medical- and patient-oriented studies. Controls $\mathrm{N}=2796$ were drawn from the Finrisk 2002 and 2007 projects. Coverage of the general population for H1N1 influenza-A Pandemrix ${ }^{\circledR}$ vaccination was $48 \%{ }^{5,25}$.

Genotyping and Imputation

HLA alleles were imputed using HIBAG and a White specific reference panel ${ }^{26}$. Posterior probabilities were high: over 95\% for each HLA gene. Positivity for DQB1*06:02 was verified directly using a panel of sequence-specific oligonucleotide probes ${ }^{27}$. Genotyping was performed at the Finnish Genome Center using Human Core Exome chip (Illumina, San Diego, CA, USA) at Finnish Institute for Molecular Medicine (FIMM) technology center genotyping unit or using Axiom EUR (Affymetrix, Santa Clara, CA, USA) at the University of California, San Francisco genotyping Unit. Quality Control (QC) for relatedness and population stratification was performed. Individuals and markers with of $>0.95$ missing values and markers with HardyWeinberg equilibrium $\mathrm{P}<0.000001$ in controls were removed $(\mathrm{N}=149$ controls and 1 case were removed due to relatedness, population stratification based on PCA, or low genotyping rate). Genotyping rate was $99.8 \%$ in the remaining individuals. Finland specific Sequencing Initiative Suomi (SISu) with 2000 whole genome and 5000 exomes was used as a reference for SNP imputation ${ }^{28,29}$. Pre-phasing was performed using SHAPEIT ${ }^{30}$ and imputed to whole genome coverage using IMPUTE2 ${ }^{31}$.

$\underline{\text { Statistical analysis }}$

We performed a frequentist association study using SNPTEST ${ }^{32}$ adjusting for $H L A D Q B 1 * 06: 02$ dosage and the first four principal components to adjust for population stratification. Results were compared to analyses without adjustment in addition to analyses stratifying controls to include only $D Q B 1 * 06: 02$ positive individuals. To estimate genetic load, we used a multilocus genetic risk score calculation, where the previously reported leading variants at each locus were chosen and weighted by previously reported odds ratio (OR) ${ }^{20-23}$ using PLINK v1.9 ${ }^{33}$ and R version 3.2.2 (2015-08-14). 


\section{Results}

Multilocus genetic risk score associates strongly with vaccination-related narcolepsy

We defined significant association at the HLA locus with vaccination-related narcolepsy $\left(\mathrm{P}=4.86^{*} 10^{-44}\right)$. Considering presence of $D Q B 1 * 06: 02$ in all cases and known increased predisposition with homozygosity ${ }^{34}$, the remaining analyses were adjusted for the number of $D Q B 1 * 06: 02$ alleles.

Altogether seven genomic regions for narcolepsy have been established to date: TRA, TRB, IFNAR1, P2RY11, TNFSF4, ZNF365 and CTSH, with the T cell receptor having the highest predisposition with an OR $>1.6$, across ethnic groups ${ }^{20-23}$. We examined whether the individual known leading risk variants (Table 1 and Figure 1) or the polygenic risk score (Figure 1) from these variants associated with narcolepsy. Some of the individual risk alleles (TRA, CTSH and $P 2 R Y 11$ ) and the weighted genetic risk score as a whole had substantial predictive power to define vaccination-triggered narcolepsy case vs. control status. The median number of risk alleles was 4 in controls and 6 in cases (Figure 1). There was a strong association of the risk score in the narcolepsy vs. control analysis using the 7 risk SNPs: $\mathrm{p}=0.0093$ and $\mathrm{p}=0.023$ when analyzed against $D Q B 1 * 06: 02$ positive controls, and $\mathrm{p}<2.2 * 10^{-16}$ when $D Q B 1 * 06: 02$ was included in the model. For predictive power when the cutoff for risk score was set to 0.71 (intercept based on ROC cost-function, optimization to limit false negatives) we could see strong predictive power with 73\% accuracy for defining narcolepsy from controls (Figure 1D). The false negative discovery rate was $4.9 \%$ with only four cases (4/81) with narcolepsy in the low risk category. On the other hand, $16.6 \%$ of controls (495/2796) were assigned in the high risk category, which is substantially clearer separation than with $D Q B 1 * 06: 02$ alone, as $28 \%$ in the control group carried this allele, yielding high specificity and sensitivity of this genetic risk score model with AUC of 0.88 (Figure 1H). The alleles contributing most to the risk score were HLA $D Q B 1 * 06: 02, T R A, P 2 R Y 11$ and $C T S H$. Single SNP level associations were seen with TRA; rs1 $154155\left(\mathrm{OR}=2.150\right.$ [1.524-3.033], $\mathrm{P}=9.61 * 10^{-05)}$ and $P 2 R Y 11$; rs $1551570(\mathrm{OR}=1.285$ [0.9141.807] $\mathrm{P}=0.0238$ ) (Figure $1 \mathrm{G})$.

Common variants affect disease risk in vaccination-related narcolepsy

We finally examined, whether distinct genetic variants would underlie vaccination-related narcolepsy. In the full GWAS, three loci close to genome-wide significance level appeared: notably, a highly protective intronic variant in $I L 12 R B 1$, which was present in $1.4 \%$ of cases vs. $11 \%$ of controls (rs17885060, OR=0.106 [0.026-0.43], P=2.93*10 ${ }^{-7}$, Figure S1, Table S1). In addition, a SNP at $Z X D C$, a gene required for HLA class II transcription, was among the top variants (rs11715293 $\mathrm{P}=7.06 * 10^{-7}, \mathrm{OR}=3.33[2.14-5.20]$, as were variants at $R B M 47$ $\left(\mathrm{OR}=2.74[1.92-3.91], \mathrm{P}=1.82 * 10^{-7}\right)$ and SORBS2 $\left(\mathrm{OR}=2.14[1.50-3.07], \mathrm{P}=3.30^{*} 10^{-7}\right)$. Although not meeting criteria for GWAS significance, the fact that 3 of the 4 associated variants are in known immune regulatory genes was notable. Further, analysis of the biological consequences of these variants defined that the rs 11715293 variant on $Z X D C$ is a STAT binding site and the risk allele was an eQTL for increased $Z X D C$ expression in whole blood $\left(\mathrm{Z}=8.23, \mathrm{P}=1.84 * 10^{-16}\right)^{35,36}$. For $I L 12 R B 1$, the most likely causative variant is the SNP with second-strongest association at the IL12RB1 locus rs3761041 (LD r ${ }^{2}=1$ with $\mathrm{rs} 17885060$ ). This variant is in interferon binding site, and an enhancer and H4K3me1 methylation site in the thymus and on T-cells, based on Encode methylation data and HaploReg ${ }^{36}$.

\section{Discussion}


In this study we discovered that previously reported narcolepsy risk variants could distinguish controls from cases of narcolepsy that have developed the disease following pandemic H1N1 vaccination, specifically with the Pandemrix ${ }^{\circledR}$ brand vaccine. This was established in a new, independent sample of cases that had not been tested for these variants. Everyone with narcolepsy was $H L A-D Q B 1 * 06: 02$ positive ${ }^{17}$. However, the addition of the 7 other known risk variants substantially improved separation between cases and controls. For comparison, the HLA$D Q B 1 * 06: 02$ allele is present in $28 \%$ of controls while only $17 \%$ of controls have a predictive score higher than the optimal threshold for predicting narcolepsy. Furthermore, using this clinically relevant threshold, fewer than $4.9 \%$ of narcolepsy cases were estimated to be within the control group resulting in $73 \%$ overall accuracy and high sensitivity and specificity. This is important to ensure potential cases of vaccine-inducible narcolepsy are not missed.

The resolution of separation with a genetic risk score is surprising and substantially large. In current medical practice $H L A D Q B 1 * 06: 02$ is often used as a tool to help with the diagnosis of narcolepsy type 1 . Using the genetic risk score that we built may provide higher resolution in defining those with genetic load. From a clinical standpoint, this is highly valuable, as a polygenic risk score may help us identify individuals that are in risk for developing this permanent and devastating disease.

The fact that large genetic effects were discovered with common variants, together with the fact most $(75 \%)$ twins are discordant for narcolepsy ${ }^{37}$, suggests that a combination of specific environmental triggers for narcolepsy must occur at the right time in combination with genetic predisposition. Given that only 1 in 16,000 vaccinated children ever developed narcolepsy (versus $\sim 0.71$ for 1000,000 at baseline) in Finland ${ }^{5}$, the environmental exposure alone appears insufficient to trigger the elevated narcolepsy incidence noted during the Pandemrix ${ }^{\circledR}$ vaccination campaign. Even in subjects with a genetic threshold above our cut off threshold, only 4 of the 10,000 of highly predisposed subjects would develop narcolepsy following Pandemrix ${ }^{\circledR}$. Clearly, although Pandemrix ${ }^{\circledR}$ was a strong trigger in this population, most predisposed patients never developed the disease, possibly explaining why risk varies substantially following vaccination or $\mathrm{H} 1 \mathrm{~N} 1$ infections in various countries ${ }^{7-10,12,13,24,25}$. The other, environmental, immunological, and genetic factors that contribute to the risk of vaccine-related narcolepsy remain unknown.

Of special interest was the fact that two of the individual risk variants (TRA and P2RY11) independently associated with vaccination-related narcolepsy ${ }^{22,23}$. Using all previously associated markers, we built a risk score estimating total genetic load and this score had strong independent effects in vaccination-related narcolepsy cases. Importantly, the effect size for TRA was higher in vaccination-related narcolepsy, with $\mathrm{OR}>2.5$, compared to $\mathrm{OR}=1.6$ in earlier studies ${ }^{20-22}$, which did not focus on vaccine-related narcolepsy. The association with TRA was also observed in a smaller post-vaccination study reported in Sweden, suggesting differential genetic predisposition for various, known, narcolepsy-associated SNPs ${ }^{24}$.

Additionally, this study provides insights that may be specific to the pathogenesis of vaccinationrelated narcolepsy. First, when examining the association of variants that may be potentially specific for vaccination related narcolepsy, we found protective variants in ILI2RBI that were nearly absent in individuals with narcolepsy, with allele frequency of $1.4 \%$ in cases but over $11 \%$ of controls. This is likely significant as prior studies have shown and replicated the findings of increased measles and pertussis toxin vaccine responses in subjects carrying specific IL12RBI receptor and $I L 12$ polymorphisms ${ }^{38-40}$. Furthermore, we found 3 additional loci nearing GWAS significance (RBM47, SORBS2 and ZXDC), one of which, a low frequency variant in transcription factor $Z X D C$ is functionally relevant to narcolepsy pathogenesis, as it is known to increase the expression of HLA class II alleles, such as $D Q B I^{*} 06: 02^{41}$. This variant, rs11715293, was highly 
enriched in cases (OR>3). The biological consequences of both the IL12RBI and ZXDC variants are interesting and likely involve increased antigen presentation to $\mathrm{CD} 4^{+} \mathrm{T}$ cells in vaccinerelated cases, similar to TRA and P2YRI1. However, although functional evidence suggests that ZXDC may affect the predisposition to vaccination-related narcolepsy this finding, as well as those of RBM47 and SORBS2, would need to be confirmed through replication in a larger sample set of vaccination-related narcolepsy cases in comparison to narcolepsy not related to vaccination.

\section{Acknowledgements and Funding}

We wish to thank Anne Huutoniemi and Minttu Sauramo for sample and data collection and coordination. This work has been supported by The EU FP7 under grant agreements nr. 313010 (BBMRI-LPC), no. 305280 (MIMOmics), and HZ2020 633589 (Ageing with Elegans), The Finnish Academy grant no. 269517 The Yrjö Jahnsson Foundation (for MP). Finnish Cultural Foundation, Sigrid Juselius Foundation, Orion Research Foundation, Jalmari and Rauha Ahokas Foundation and Academy of Finland (HMO). NIHP50-NS023724-19A1 (EM). NIHT32 HL110952 05 (LDS).

\section{References}

1. Hublin, C., Partinen, M., Kaprio, J., Koskenvuo, M. \& Guilleminault, C. Epidemiology of narcolepsy. Sleep 17, S7-12 (1994).

2. Ohayon, M.M., Priest, R.G., Zulley, J., Smirne, S. \& Paiva, T. Prevalence of narcolepsy symptomatology and diagnosis in the European general population. Neurology 58, 1826-33 (2002).

3. Silber, M.H., Krahn, L.E., Olson, E.J. \& Pankratz, V.S. The epidemiology of narcolepsy in Olmsted County, Minnesota: a population-based study. Sleep 25, 197-202 (2002).

4. Peyron, C. et al. A mutation in a case of early onset narcolepsy and a generalized absence of hypocretin peptides in human narcoleptic brains. Nat Med 6, 991-7 (2000).

5. Nohynek, H. et al. AS03 adjuvanted AH1N1 vaccine associated with an abrupt increase in the incidence of childhood narcolepsy in Finland. PLoS One 7, e33536 (2012).

6. Persson, I. et al. Risks of neurological and immune-related diseases, including narcolepsy, after vaccination with Pandemrix: a population- and registrybased cohort study with over 2 years of follow-up. J Intern Med 275, 172-90 (2014).

7. Partinen, M. et al. Increased incidence and clinical picture of childhood narcolepsy following the $2009 \mathrm{H} 1 \mathrm{~N} 1$ pandemic vaccination campaign in Finland. PLoS One 7, e33723 (2012).

8. O'Flanagan, D. et al. Investigation of an association between onset of narcolepsy and vaccination with pandemic influenza vaccine, Ireland April 2009-December 2010. Euro Surveill 19, 15-25 (2014).

9. Heier, M.S. et al. Incidence of narcolepsy in Norwegian children and adolescents after vaccination against H1N1 influenza A. Sleep Med 14, 867-71 (2013).

10. Dauvilliers, Y. et al. Increased risk of narcolepsy in children and adults after pandemic H1N1 vaccination in France. Brain 136, 2486-96 (2013). 
11. Winstone, A.M. et al. Clinical features of narcolepsy in children vaccinated with AS03 adjuvanted pandemic A/H1N1 2009 influenza vaccine in England. Dev Med Child Neurol 56, 1117-23 (2014).

12. Gadroen, K. et al. Patterns of spontaneous reports on narcolepsy following administration of pandemic influenza vaccine; a case series of individual case safety reports in Eudravigilance. Vaccine 34, 4892-7 (2016).

13. Montplaisir, J. et al. Risk of narcolepsy associated with inactivated adjuvanted (AS03) A/H1N1 (2009) pandemic influenza vaccine in Quebec. PLoS One 9, e108489 (2014).

14. Ambati, A. et al. Increased beta-haemolytic group A streptococcal M6 serotype and streptodornase B-specific cellular immune responses in Swedish narcolepsy cases. J Intern Med 278, 264-76 (2015).

15. Han, F. et al. Narcolepsy onset is seasonal and increased following the 2009 H1N1 pandemic in China. Ann Neurol 70, $410-7$ (2011).

16. Aran, A. et al. Elevated anti-streptococcal antibodies in patients with recent narcolepsy onset. Sleep 32, 979-83 (2009).

17. Mignot, E. et al. Complex HLA-DR and -DQ interactions confer risk of narcolepsy-cataplexy in three ethnic groups. Am J Hum Genet 68, 686-99 (2001).

18. Ollila, H.M. et al. HLA-DPB1 and HLA class I confer risk of and protection from narcolepsy. Am J Hum Genet 96, 136-46 (2015).

19. Tafti, M. et al. DQB1 Locus Alone Explains Most of the Risk and Protection in Narcolepsy with Cataplexy in Europe. Sleep 37, 19-25 (2014).

20. Han, F. et al. Genome wide analysis of narcolepsy in China implicates novel immune loci and reveals changes in association prior to versus after the 2009 H1N1 influenza pandemic. PLoS Genet 9, e1003880 (2013).

21. Faraco, J. et al. ImmunoChip study implicates antigen presentation to T cells in narcolepsy. PLoS Genet 9, e1003270 (2013).

22. Hallmayer, J. et al. Narcolepsy is strongly associated with the T-cell receptor alpha locus. Nat Genet 41, 708-11 (2009).

23. Kornum, B.R. et al. Common variants in P2RY11 are associated with narcolepsy. Nat Genet 43, 66-71 (2011).

24. Bomfim, I.L. et al. The immunogenetics of narcolepsy associated with A(H1N1)pdm09 vaccination (Pandemrix) supports a potent geneenvironment interaction. Genes Immun 18, 75-81 (2017).

25. Gil Cuesta, J. et al. Pandemic vaccination strategies and influenza severe outcomes during the influenza A(H1N1)pdm09 pandemic and the postpandemic influenza season: the Nordic experience. Euro Surveill 21(2016).

26. Zheng, X. et al. HIBAG--HLA genotype imputation with attribute bagging. Pharmacogenomics J 14, 192-200 (2014).

27. Hermann, R. et al. HLA DR-DQ-encoded genetic determinants of childhoodonset type 1 diabetes in Finland: an analysis of 622 nuclear families. Tissue Antigens 62, 162-9 (2003).

28. Cousminer, D.L. et al. Targeted resequencing of the pericentromere of chromosome 2 linked to constitutional delay of growth and puberty. PLoS One 10, e0128524 (2015). 
29. Lim, E.T. et al. Distribution and medical impact of loss-of-function variants in the Finnish founder population. PLoS Genet 10, e1004494 (2014).

30. Delaneau, 0., Marchini, J. \& Zagury, J.F. A linear complexity phasing method for thousands of genomes. Nat Methods 9, 179-81 (2011).

31. Howie, B.N., Donnelly, P. \& Marchini, J. A flexible and accurate genotype imputation method for the next generation of genome-wide association studies. PLoS Genet 5, e1000529 (2009).

32. Wellcome Trust Case Control, C. Genome-wide association study of 14,000 cases of seven common diseases and 3,000 shared controls. Nature 447, 66178 (2007).

33. Chang, C.C. et al. Second-generation PLINK: rising to the challenge of larger and richer datasets. Gigascience 4, 7 (2015).

34. Pelin, Z., Guilleminault, C., Risch, N., Grumet, F.C. \& Mignot, E. HLADQB1*0602 homozygosity increases relative risk for narcolepsy but not disease severity in two ethnic groups. US Modafinil in Narcolepsy Multicenter Study Group. Tissue Antigens 51, 96-100 (1998).

35. Westra, H.J. et al. Systematic identification of trans eQTLs as putative drivers of known disease associations. Nat Genet 45, 1238-43 (2013).

36. Kheradpour, P. \& Kellis, M. Systematic discovery and characterization of regulatory motifs in ENCODE TF binding experiments. Nucleic Acids Res 42, 2976-87 (2014).

37. Mignot, E. Genetic and familial aspects of narcolepsy. Neurology 50, S16-22 (1998).

38. Grondahl-Yli-Hannuksela, K., Vahlberg, T., Ilonen, J., Mertsola, J. \& He, Q. Polymorphism of IL-10 gene promoter region: association with T cell proliferative responses after acellular pertussis vaccination in adults. Immunogenetics 68, 733-41 (2016).

39. Haralambieva, I.H. et al. Associations between single nucleotide polymorphisms and haplotypes in cytokine and cytokine receptor genes and immunity to measles vaccination. Vaccine 29, 7883-95 (2011).

40. White, S.J. et al. Replication of associations between cytokine and cytokine receptor single nucleotide polymorphisms and measles-specific adaptive immunophenotypic extremes. Hum Immunol 73, 636-40 (2012).

41. Aleksandrova, A., Galkin, O., Koneni, R. \& Fontes, J.D. An N- and C-terminal truncated isoform of zinc finger X-linked duplicated $C$ protein represses $\mathrm{MHC}$ class II transcription. Mol Cell Biochem 337, 1-7 (2010).

\section{Figure legends}

Figure 1. (A) Genetic risk score without $H L A D Q B 1 * 06: 02$ associates with narcolepsy case control status. (B) The separation is seen also when stratifying the controls to be $D Q B 1 * 06: 02$ positive (C) By including both previously discovered genetic risk factors and HLA DQB1 *06:02, strong predictive value is seen with only $4 / 81$ individuals with narcolepsy (4.9\%) false negative 
and 495/2796 controls (16.6\%) false positive; $28 \%$ of controls and 100\% narcolepsy cases were positive for the $D Q B 1 * 06: 02$ marker. (D) The categorization accuracy of the genetic risk score was $73 \%$. The median number of risk alleles (E) in cases was 6 vs. (F) 4 in the controls. (G) Association of individual risk variants from previous GWAS studies in vaccination-related narcolepsy. (H) ROC curve analysis confirms that the overall score had high sensitivity and specificity with AUC 0.88.

Table 1. Replication of individual risk variants for narcolepsy and summarized effects

\begin{tabular}{|l|l|l|l|r|l|}
\hline Gene & CHR - bp & Reported SNP & Coded allele & P-value & OR \\
\hline TRA & $14-23002684$ & rs1154155 & G & $9.61 \mathrm{E}-05$ & $2.150[1.524-3.033]$ \\
\hline TRB & $7-142039520$ & $\mathrm{rs} 9648789$ & $\mathrm{~T}$ & 0.192 & $0.810[0.558-1.176]$ \\
\hline CTSH & $15-79234957$ & $\mathrm{rs34593439}$ & $\mathrm{A}$ & $0.1261 .823[1.096-3.032]$ \\
\hline TNFSF4/OX40L/CD252 & $1-173131908$ & $\mathrm{rs} 7553711$ & $\mathrm{C}$ & 0.509 & $1.084[0.765-1.535]$ \\
\hline ZNF365 & $10-64391375$ & $\mathrm{rs} 10995245$ & $\mathrm{~A}$ & 0.443 & $0.943[0.669-1.329]$ \\
\hline P2RY11 & $19-10226052$ & $\mathrm{rs} 2305795$ & $\mathrm{~A}$ & $0.0351 .277[0.908-1.795]$ \\
\hline IL10RB-IFNAR1 & $21-34689253$ & $\mathrm{rs} 2834188$ & $\mathrm{G}$ & 0.793 & $0.979[0.659-1.455]$ \\
\hline DQB1*06:02 & $6-32000000$ & DQB1*06:02 & Present & $2^{*} 10^{-54}$ & INF \\
\hline
\end{tabular}

odds ratio (OR), base pair (bp), CHR (chromosome) 
A Genetic risk score without DQB1*06:02

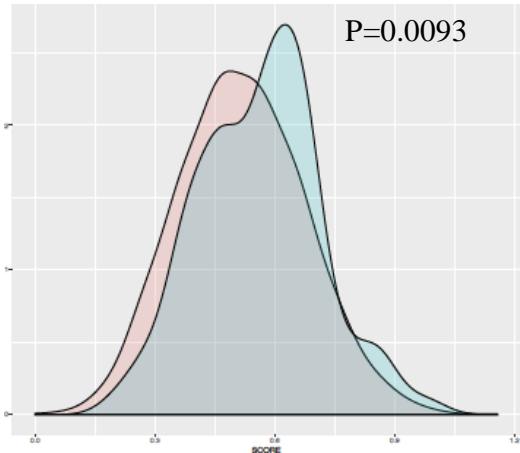

E N risk alleles in cases (DQB1*06:02 not included)

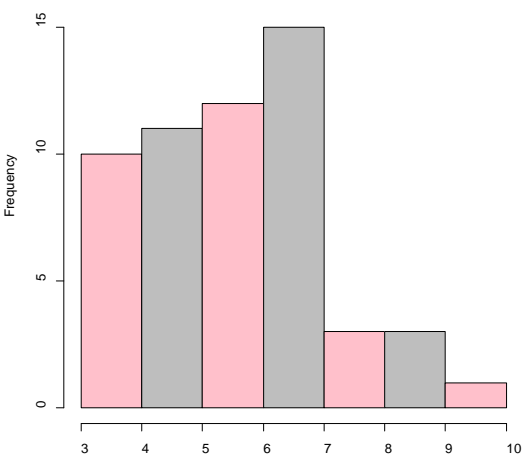

B Genetic risk score in DQB1*06:02 carriers

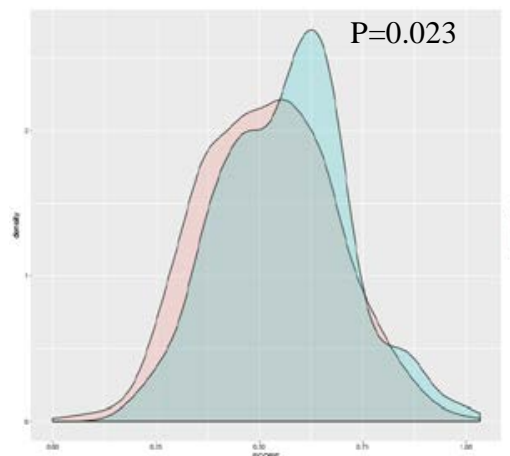

F N risk alleles in controls (DQB1*06:02 not included)

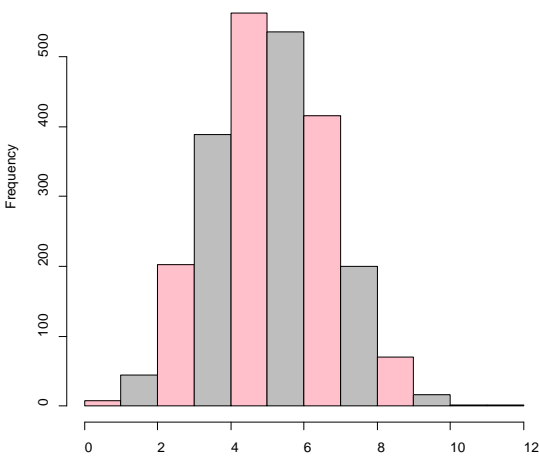

C Genetic risk score with DQB1*06:02

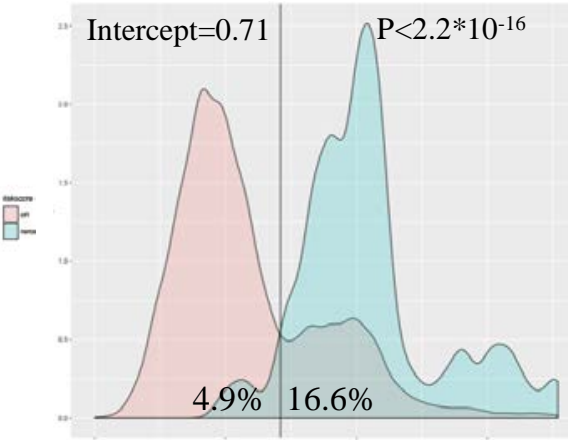

G Effect of each previously found risk variant

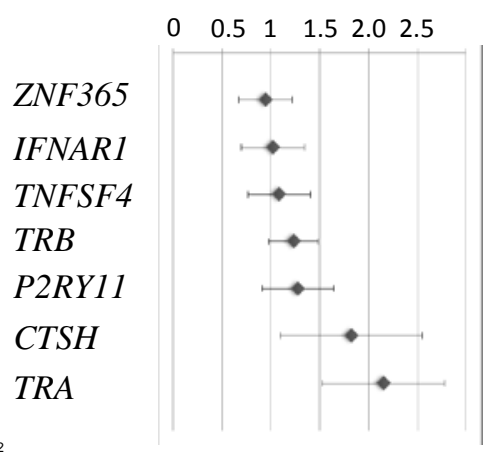

D ROC Accuracy

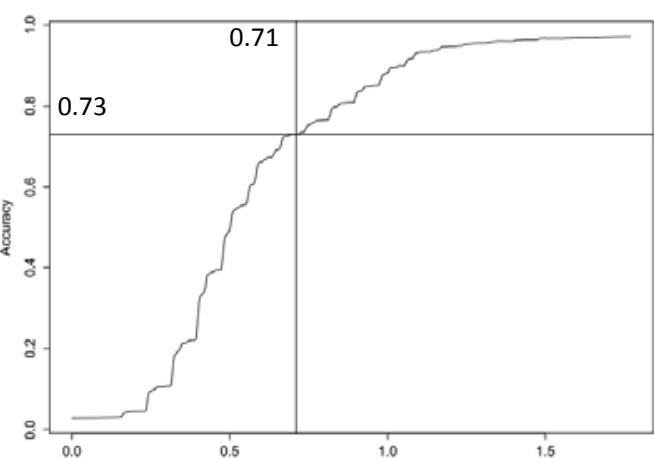

H ROC Sensitivity vs. Specificity

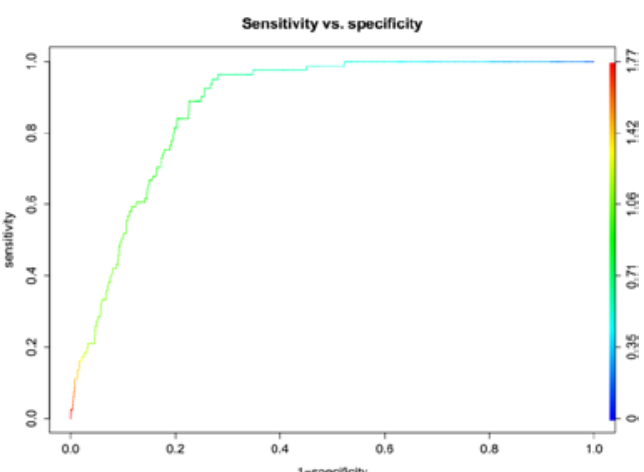

